\title{
Performance of the Graybill-Deal Estimator via Pitman Closeness Criterion
}

\author{
Keyu Nie, Bikas.K. Sinha tand A.S. Hedayat \\ Department of Mathematics, Statistics, and Computer Science \\ University of Illinois at Chicago
}

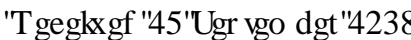

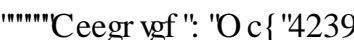

\begin{abstract}
Pitman closeness criterion is a coverage probability-based criterion to examine the relative performances of estimators. Usually, the performance of the standard Graybill-Deal estimator of the common mean has been examined with respect to the mean squared error (variance). In this study we examine its performance with respect to the Pitman closeness criterion. Specifically, we compare a $p$-source based Graybill-Deal estimator against its $q$-source based competitors for $q(<p)$-source subsets of $p$-source data. The key references to this paper are [5] and [7].
\end{abstract}

Keywords: Graybill-Deal Estimator; Pitman Closeness; Meta Analysis.

2000 Mathematics Subject Classification: 62F30

\section{Introduction}

We consider the problem of estimation of the common mean shared by several independent normal populations with unknown and most likely unequal variances. Generally, we have $p$ independent sources with distributions $N\left(\mu, \sigma_{i}^{2}\right), i=1,2, \ldots, p$. Also, let $\bar{x}_{i}$ and $s_{i}^{2}$ be sample mean and sample variance, respectively; and $s_{\bar{x}_{i}}^{2}=s_{i}^{2} / n_{i}, \sigma_{\bar{x}_{i}}^{2}=\sigma_{i}^{2} / n_{i}$, where $n_{i}$ is the sample size available from the $i^{\text {th }}$ source, $i=1,2, \ldots, p$.

[1] introduced their estimator $\hat{\mu}_{G D \mid 2}$ for $p=2$ sources, and claimed that $\hat{\mu}_{G D \mid 2}$ was preferable to both sample means with respect to the criteria of mean square error, if and only if sample sizes $n_{1}$ and $n_{2}$ were moderate enough ( $\left.\geq 11\right)$, which was corrected by [4] as $\left(n_{1} \geq 11, n_{2} \geq 11\right)$, $\left(n_{1}=10, n_{2} \geq 19\right)$ or $\left(n_{1} \geq 19, n_{2}=10\right)$. Subsequently this result was extended by [3] to include $p$ independent sources. They compared the Graybill-Deal estimator (GDE) of combining $p$ sources

$$
\hat{\mu}_{G D \mid p}=\sum_{i=1}^{p} \frac{\frac{\bar{x}_{i}}{s_{\bar{x}_{i}}^{2}}}{\sum_{i=1}^{p} \frac{1}{s_{\bar{x}_{i}}^{2}}}
$$

\footnotetext{
*keyunie@gmail.com

†bikassinha1946@gmail.com

†hedayat@uic.edu
} 
with sample means $\bar{x}_{i}, i=1,2, \ldots, p$, and found that $\hat{\mu}_{G D \mid p}$ had a smaller variance than each sample mean under the same condition as [1]; that is, either $n_{i}>10$ for $i=1,2, \ldots, p$, or $n_{i}=10$ for some $i$, and $n_{j}>18(i, j=1,2, \ldots, p$ for each $j \neq i)$. [8] showed that $\hat{\mu}_{G D \mid p}$ dominated any $\hat{\mu}_{G D \mid q}$ of $q$ $(<p)$ sub-sources, under the same condition as in [3], with respect to mean square criterion.

Here we shall compare $\hat{\mu}_{G D \mid p}$ with $\hat{\mu}_{G D \mid q}$ by employing Pitman closeness criterion, which was introduced by [6]: We say estimator $\hat{\mu}_{1}$ is better (Pitman-closer) than $\hat{\mu}_{2}$ for the estimation of the parameter $\mu$ if and only if $P\left\{\left|\hat{\mu}_{1}-\mu\right| \leq\left|\hat{\mu}_{2}-\mu\right|\right\} \geq 1 / 2$. Pitman-closeness criterion is a coverage probability-based criterion and has nothing to do with the loss function. It provides another angle in the sense of 'one-to-one' comparison measuring the performance efficiency of GDE.

Using the Pitman-closeness criterion, [5] and [7] established that for $p=2$, a necessary and sufficient condition for

$$
P\left\{\left|\hat{\mu}_{G D \mid 2}-\mu\right| \leq\left|\bar{x}_{i}-\mu\right|\right\} \geq 1 / 2,
$$

to hold uniformly in $\left(\mu, \sigma_{\bar{x}_{1}}^{2}, \sigma_{\bar{x}_{2}}^{2}\right)$ is that $m_{i}=n_{i}-1 \geq 4$ for each $i=1,2$. [7] further established that

$$
P\left\{\left|\hat{\mu}_{G D \mid p}-\mu\right| \leq\left|\bar{x}_{i}-\mu\right|\right\} \geq 1 / 2,
$$

for all $i=1,2, \ldots, p$ and uniformly in $\left(\mu, \sigma_{\bar{x}_{1}}^{2}, \sigma_{\bar{x}_{2}}^{2}, \ldots, \sigma_{\bar{x}_{p}}^{2}\right)$, if and only if

$$
2 E\left\{\left(\sum_{j=1, j \neq i}^{p} \sigma_{\bar{x}_{j}}^{-2} Y_{j}^{2}\right)^{-1 / 2}\left(\sum_{j=1, j \neq i}^{p} \sigma_{\bar{x}_{j}}^{-2} Y_{j}\right)\right\} \leq E\left\{\left(\sum_{j=1, j \neq i}^{p} \sigma_{\bar{x}_{j}}^{-2} Y_{j}^{2}\right)^{1 / 2}\right\},
$$

holds for all $i=1,2, \ldots, p$ and uniformly in $\left(\sigma_{\bar{x}_{1}}^{2}, \sigma_{\bar{x}_{2}}^{2}, \ldots, \sigma_{\bar{x}_{p}}^{2}\right)$, where $Y_{j}$ 's are independently distributed as $\frac{m_{j}}{\chi^{2}\left(m_{j}\right)}$ and $m_{j}=n_{j}-1$, for $j=1,2, \ldots, p$. [7] also gave a sufficient condition by showing that inequality (1.1) holds, for any $i=1,2, \ldots, p$, if

$$
1-8 m_{k}^{-1}+4\left(\sum_{j=1, j \neq i}^{p} m_{j}\right)^{-1} \geq 0
$$

for all $k$ in $\{1,2, \ldots, i-1, i+1, \ldots, p\}$.

In this paper we show that $\hat{\mu}_{G D \mid p}$ is a Pitman-closer estimator than $\hat{\mu}_{G D \mid q}$, the GDE of any $q(<p)$ sub-sources, in the sense of Pitman closeness criteria for all $p>2$. Without loss of generality, our results are presented by comparing with initial $q$ sub-sources, although for any other $q$ sub-sources, we will obtain similar results.

We provide several sufficient conditions [including the inequality (1.1) (suitably modified) which holds uniformly in $\left(\sigma_{\bar{x}_{1}}^{2}, \sigma_{\bar{x}_{2}}^{2}, \ldots, \sigma_{\bar{x}_{p}}^{2}\right)$ for all $q$ sub-sources]. We also provide a necessary condition towards this.

The remainder of this paper is structured as follows. Section 2 describes all the lemmas and preparation for the main results. Section 3 provides a necessary condition when combining all $p$ sources is preferred. Section 4 presents the corresponding sufficient condition and several corollaries. Section 5 discusses the sample size requirement. Section 6 concludes the paper.

\section{Notations and Lemmas}

Before introducing our main results, we first introduce the following notations and lemmas. 
We borrow the definition from [7]:

Let

$$
Y_{i}=\sigma_{\bar{x}_{i}}^{2} s_{\bar{x}_{i}}^{-2} \sim \frac{m_{i}}{\chi^{2}\left(m_{i}\right)}, \text { for } i=1,2, \ldots, p,
$$

where $Y_{i}$ 's are independently distributed and $m_{i}=n_{i}-1$ is the degrees of freedom. Obviously we have $E\left(Y_{i}^{-1}\right)=1$.

Let $p=q+r$ and define:

$$
\begin{aligned}
U_{q} & =\left(\sum_{i=1}^{q} \sigma_{\bar{x}_{i}}^{2} s_{\bar{x}_{i}}^{-4}\right)^{-\frac{1}{2}}\left(\sum_{i=1}^{q} s_{\bar{x}_{i}}^{-2}\right)=\left(\sum_{i=1}^{q} \sigma_{\bar{x}_{i}}^{-2} Y_{i}^{2}\right)^{-\frac{1}{2}}\left(\sum_{i=1}^{q} \sigma_{\bar{x}_{i}}^{-2} Y_{i}\right), \\
U_{r} & =\left(\sum_{i=q+1}^{q+r} \sigma_{\bar{x}_{i}}^{2} s_{\bar{x}_{i}}^{-4}\right)^{-\frac{1}{2}}\left(\sum_{i=q+1}^{q+r} s_{\bar{x}_{i}}^{-2}\right)=\left(\sum_{i=q+1}^{q+r} \sigma_{\bar{x}_{i}}^{-2} Y_{i}^{2}\right)^{-\frac{1}{2}}\left(\sum_{i=q+1}^{q+r} \sigma_{\bar{x}_{i}}^{-2} Y_{i}\right), \\
V_{q} & =\left(\sum_{i=1}^{q} \sigma_{\bar{x}_{i}}^{2} s_{\bar{x}_{i}}^{-4}\right)^{-\frac{1}{2}}=\left(\sum_{i=1}^{q} \sigma_{\bar{x}_{i}}^{-2} Y_{i}^{2}\right)^{-\frac{1}{2}}, \\
V_{r} & =\left(\sum_{i=q+1}^{q+r} \sigma_{\bar{x}_{i}}^{2} s_{\bar{x}_{i}}^{-4}\right)^{-\frac{1}{2}}=\left(\sum_{i=q+1}^{q+r} \sigma_{\bar{x}_{i}}^{-2} Y_{i}^{2}\right)^{-\frac{1}{2}} .
\end{aligned}
$$

$\left(U_{q}, V_{q}\right)$ and $\left(U_{r}, V_{r}\right)$ are mutually independent. As we mentioned before, $U_{q}$ and $V_{q}$ represented the initial $q$ subgroups, and hence $U_{r}$ and $V_{r}$ represented the remaining $r$ subgroups.

Lemma 2.1. $\sum_{i=1}^{h} \sigma_{\bar{x}_{i}}^{-2} \geq U_{h}^{2} \geq \min _{i=1,2, \ldots, h}\left\{\sigma_{\bar{x}_{i}}^{-2}\right\}$ holds uniformly in $\left(\sigma_{\bar{x}_{1}}^{2}, \sigma_{\bar{x}_{2}}^{2}, \ldots, \sigma_{\bar{x}_{h}}^{2}\right)$.

Proof. Due to Cauchy-Schwarz inequality, we have:

$$
\begin{aligned}
U_{h}^{2} & =\left(\sum_{i=1}^{h} \sigma_{\bar{x}_{i}}^{-2} Y_{i}^{2}\right)^{-1}\left(\sum_{i=1}^{h} \sigma_{\bar{x}_{i}}^{-2} Y_{i}\right)^{2} \\
& \leq \sum_{i=1}^{h} \sigma_{\bar{x}_{i}}^{-2} .
\end{aligned}
$$

On the other side,

$$
\begin{aligned}
U_{h}^{2} & =\left(\sum_{i=1}^{h} \sigma_{\bar{x}_{i}}^{-2} Y_{i}^{2}\right)^{-1}\left(\sum_{i=1}^{h} \sigma_{\bar{x}_{i}}^{-2} Y_{i}\right)^{2} \\
& \geq\left(\sum_{i=1}^{h} \sigma_{\bar{x}_{i}}^{-2} Y_{i}^{2}\right)^{-1}\left(\sum_{i=1}^{h} \sigma_{\bar{x}_{i}}^{-4} Y_{i}^{2}\right) \\
& =\sum_{i=1}^{h} \frac{\sigma_{\bar{x}_{i}}^{-2} Y_{i}^{2}}{\sum_{i=1}^{h} \sigma_{\bar{x}_{i}}^{-2} Y_{i}^{2}} \sigma_{\bar{x}_{i}}^{-2} \\
& \geq \min _{i=1,2, \ldots, h}\left\{\sigma_{\bar{x}_{i}}^{-2}\right\} .
\end{aligned}
$$

Lemma 2.2. The inequality $E\left\{U_{h}^{-1}\right\} \geq\left(\sum_{i=1}^{h} \sigma_{\bar{x}_{i}}^{-2}\right)^{-1 / 2} \geq E\left\{V_{h}\right\}$ holds uniformly in $\left(\sigma_{\bar{x}_{1}}^{2}, \sigma_{\bar{x}_{2}}^{2}, \ldots, \sigma_{\bar{x}_{h}}^{2}\right)$. 
Proof. Due to the fact:

$$
\begin{aligned}
E\left\{U_{h}^{-1}\right\} & =E\left\{\left(\sum_{i=1}^{h} \sigma_{\bar{x}_{i}}^{-2} Y_{i}^{2}\right)^{1 / 2}\left(\sum_{i=1}^{h} \sigma_{\bar{x}_{i}}^{-2} Y_{i}\right)^{-1}\right\} \\
& \geq\left(\sum_{i=1}^{h} \sigma_{\bar{x}_{i}}^{-2}\right)^{-1 / 2} \\
& =\left(\sum_{i=1}^{h} \sigma_{\bar{x}_{i}}^{-2}\right)^{-1 / 2} E\left\{\sum_{j=1}^{h} \frac{\sigma_{\bar{x}_{j}}^{-2}}{\sum_{i=1}^{h} \sigma_{\bar{x}_{i}}^{-2}} Y_{j}^{-1}\right\} \\
& =\left(\sum_{i=1}^{h} \sigma_{\bar{x}_{i}}^{-2}\right)^{-1 / 2} E\left\{\sum_{j=1}^{h} \frac{\sigma_{\bar{x}_{j}}^{-2}}{\sum_{i=1}^{h} \sigma_{\bar{x}_{i}}^{-2}}\left(Y_{j}^{2}\right)^{-1 / 2}\right\} \\
& \geq\left(\sum_{i=1}^{h} \sigma_{\bar{x}_{i}}^{-2}\right)^{-1 / 2} E\left\{\sum_{j=1}^{h} \frac{\sigma_{\bar{x}_{j}}^{-2}}{\sum_{i=1}^{h} \sigma_{\bar{x}_{i}}^{-2}} Y_{j}^{2}\right\}^{-1 / 2} \\
& =E\left\{\sum_{j=1}^{h} \sigma_{\bar{x}_{j}}^{-2} Y_{j}^{2}\right\}^{-1 / 2} \\
& =E\left\{V_{h}\right\} .
\end{aligned}
$$

The inequality (2.1) is based on Lemma 2.1 , and inequality (2.2) is due to Jensen's inequality.

Lemma 2.3. The inequality $\left(\sum_{i=1}^{h} \sigma_{\bar{x}_{i}}^{-2}\right) E\left(U_{h}^{-1}\right) \leq E\left(V_{h}^{-1}\right)$ holds uniformly in $\left(\sigma_{\bar{x}_{1}}^{2}, \sigma_{\bar{x}_{2}}^{2}, \ldots, \sigma_{\bar{x}_{h}}^{2}\right)$.

Proof. See [7].

Lemma 2.4. The probability $P\left\{\left|\hat{\mu}_{G D \mid p}-\mu\right| \leq\left|\hat{\mu}_{G D \mid q}-\mu\right|\right\}$ is $1 / \pi\left(E\left\{\arctan \left(U_{r} U_{q}^{-1}\right)\right\}+E\left\{\arctan \left(U_{r} U_{q}^{-1}+2 V_{r} V_{q}^{-1}\right)\right\}\right)$.

Proof. Without loss of generality, it is clear $\mu$ may be assumed to be 0 . As $p=q+r$, we need to compute the probability $P\left\{\left|\hat{\mu}_{G D \mid p}\right| \leq\left|\hat{\mu}_{G D \mid q}\right|\right\}$.

By applying the fact that:

$$
\hat{\mu}_{G D \mid p}=\frac{\left(\sum_{i=1}^{q} s_{\bar{x}_{i}}^{-2}\right) \hat{\mu}_{G D \mid q}+\left(\sum_{i=q+1}^{q+r} s_{\bar{x}_{i}}^{-2}\right) \hat{\mu}_{G D \mid r}}{\sum_{i=1}^{q+r} s_{\bar{x}_{i}}^{-2}},
$$

the probability $P\left\{\left|\hat{\mu}_{G D \mid p}\right| \leq\left|\hat{\mu}_{G D \mid q}\right|\right\}$ can be written as:

$$
\begin{aligned}
& P\left\{\left|\hat{\mu}_{G D \mid p}\right| \leq\left|\hat{\mu}_{G D \mid q}\right|\right\} \\
& =P\left\{\left(\hat{\mu}_{G D \mid p}\right)^{2} \leq\left(\hat{\mu}_{G D \mid q}\right)^{2}\right\} \\
& =P\left\{\left(\frac{\left(\sum_{i=1}^{q} s_{\bar{x}_{i}}^{-2}\right) \hat{\mu}_{G D \mid q}+\left(\sum_{i=q+1}^{q+r} s_{\bar{x}_{i}}^{-2}\right) \hat{\mu}_{G D \mid r}}{\sum_{i=1}^{q+r} s_{\bar{x}_{i}}^{-2}}\right)^{2} \leq\left(\hat{\mu}_{G D \mid q}\right)^{2}\right\} \\
& =P\left\{\left(\left(\sum_{i=1}^{q} s_{\bar{x}_{i}}^{-2}\right) \hat{\mu}_{G D \mid q}+\left(\sum_{i=q+1}^{q+r} s_{\bar{x}_{i}}^{-2}\right) \hat{\mu}_{G D \mid r}\right)^{2} \leq\left(\left(\sum_{i=1}^{q+r} s_{\bar{x}_{i}}^{-2}\right) \hat{\mu}_{G D \mid q}\right)^{2}\right\} \\
& =2 P\left\{\hat{\mu}_{G D \mid q}\left(1+2 \frac{\sum_{i=1}^{q} s_{\bar{x}_{i}}^{-2}}{\sum_{i=q+1}^{q+r} s_{\bar{x}_{i}}^{-2}}\right) \leq \hat{\mu}_{G D \mid r} \leq \hat{\mu}_{G D \mid q}, \hat{\mu}_{G D \mid q}>0\right\}
\end{aligned}
$$




$$
\begin{aligned}
& =P\left\{-\left(\frac{\left(\sum_{i=1}^{q} \sigma_{\bar{x}_{i}}^{2} \overline{\bar{x}}_{i}^{-4}\right)^{1 / 2}\left(\sum_{i=1}^{q} s_{\bar{x}_{i}}^{-2}\right)^{-1}}{\left(\sum_{i=q+1}^{q+r} \sigma_{\bar{x}_{i}}^{2} \overline{\bar{x}}_{i}^{-4}\right)^{1 / 2}\left(\sum_{i=q+1}^{q+r} s_{\bar{x}_{i}}^{-2}\right)^{-1}}+2 \frac{\left(\sum_{i=1}^{q} \sigma_{\bar{x}_{i}}^{2} s_{\bar{x}_{i}}^{-4}\right)^{1 / 2}}{\left(\sum_{i=q+1}^{q+r} \sigma_{\bar{x}_{i}}^{2} s_{\bar{x}_{i}}^{-4}\right)^{1 / 2}}\right)\right. \\
& \leq\left(\left(\frac{\hat{\mu}_{G D \mid r}}{\left(\sum_{i=q+1}^{q+r} \sigma_{\bar{x}_{i}}^{2} s_{\bar{x}_{i}}^{-4}\right)^{1 / 2}\left(\sum_{i=q+1}^{q+r} s_{\bar{x}_{i}}^{-2}\right)^{-1}}\right) /\left(\frac{\hat{\mu}_{G D \mid q}}{\left(\sum_{i=1}^{q} \sigma_{\bar{x}_{i}}^{2} s_{\bar{x}_{i}}^{-4}\right)^{1 / 2}\left(\sum_{i=1}^{q} s_{\bar{x}_{i}}^{-2}\right)^{-1}}\right)\right) \\
& \left.\leq \frac{\left(\sum_{i=1}^{q} \sigma_{\bar{x}_{i}}^{2} s_{\bar{x}_{i}}^{-4}\right)^{1 / 2}\left(\sum_{i=1}^{q} s_{\bar{x}_{i}}^{-2}\right)^{-1}}{\left(\sum_{i=q+1}^{q+r} \sigma_{\bar{x}_{i}}^{2} s_{\bar{x}_{i}}^{-4}\right)^{1 / 2}\left(\sum_{i=q+1}^{q+r} s_{\bar{x}_{i}}^{-2}\right)^{-1}}\right\} \\
& =P\left\{-\left(U_{r} U_{q}^{-1}+2 V_{r} V_{q}^{-1}\right) \leq \frac{\frac{\hat{\mu}_{G D \mid r}}{\left(\sum_{i=q+1}^{q+r} \sigma_{\bar{x}_{i}}^{2} s_{\bar{x}_{i}}^{-4}\right)^{1 / 2}\left(\sum_{i=q+1}^{q+r} s_{\overline{x_{i}}}^{-2}\right)^{-1}}}{\frac{\hat{\mu}_{G D \mid q}}{\left(\sum_{i=1}^{q} \sigma_{\bar{x}_{i}}^{2} s_{\bar{x}_{i}}^{-4}\right)^{1 / 2}\left(\sum_{i=1}^{q} s_{\bar{x}_{i}}^{-2}\right)^{-1}}} \leq U_{r} U_{q}^{-1}\right\} .
\end{aligned}
$$

Note that conditionally given $s_{\bar{x}_{i}}^{-2}$ for $i=1, \ldots, p$, the ratio

$$
\frac{\frac{\hat{\mu}_{G D \mid r}}{\left(\sum_{i=q+1}^{q+r} \sigma_{\bar{x}_{i}}^{2} s_{\overline{\bar{x}_{i}}}^{-4}\right)^{1 / 2}\left(\sum_{i=q+1}^{q+r} s_{\bar{x}_{i}}^{-2}\right)^{-1}}}{\frac{\hat{\mu}_{G D \mid q}}{\left(\sum_{i=1}^{q} \sigma_{\bar{x}_{i}}^{2} s_{\bar{x}_{i}}^{-4}\right)^{1 / 2}\left(\sum_{i=1}^{q} s_{\bar{x}_{i}}^{-2}\right)^{-1}}}
$$

follows a Cauchy distribution.

Therefore, the probability in Eq.(2.3), can be denoted as

$$
\begin{aligned}
\gamma & =\gamma\left(\sigma_{\bar{x}_{1}}^{2}, \sigma_{\bar{x}_{2}}^{2}, \ldots, \sigma_{\bar{x}_{p}}^{2}\right) \\
& =1 / \pi\left(E\left\{\arctan \left(U_{r} U_{q}^{-1}\right)\right\}-E\left\{\arctan \left(-U_{r} U_{q}^{-1}-2 V_{r} V_{q}^{-1}\right)\right\}\right) \\
& =1 / \pi\left(E\left\{\arctan \left(U_{r} U_{q}^{-1}\right)\right\}+E\left\{\arctan \left(U_{r} U_{q}^{-1}+2 V_{r} V_{q}^{-1}\right)\right\}\right) .
\end{aligned}
$$

\section{When is combining preferable ? A Necessary Condition}

Theorem 3.1. $P\left\{\left|\hat{\mu}_{G D \mid p}-\mu\right| \leq\left|\hat{\mu}_{G D \mid q}-\mu\right|\right\} \geq 1 / 2$ holds uniformly in $\left(\mu, \sigma_{\bar{x}_{1}}^{2}, \sigma_{\bar{x}_{2}}^{2}, \ldots, \sigma_{\bar{x}_{p}}^{2}\right)$, only if

$$
\begin{aligned}
& 2 E\left\{\left(\sum_{i=q+1}^{p} \sigma_{\bar{x}_{i}}^{-2} Y_{i}^{2}\right)^{-1 / 2}\left(\sum_{i=q+1}^{p} \sigma_{\bar{x}_{i}}^{-2} Y_{i}\right)\right\} E\left\{\left(\sum_{i=1}^{q} \sigma_{\bar{x}_{i}}^{-2} Y_{i}^{2}\right)^{1 / 2}\left(\sum_{i=1}^{q} \sigma_{\bar{x}_{i}}^{-2} Y_{i}\right)^{-1}\right\} \leq \\
& E\left\{\left(\sum_{i=q+1}^{p} \sigma_{\bar{x}_{i}}^{-2} Y_{i}^{2}\right)^{1 / 2}\right\} E\left\{\left(\sum_{i=1}^{q} \sigma_{\bar{x}_{i}}^{-2} Y_{i}^{2}\right)^{-1 / 2}\right\},
\end{aligned}
$$

which is equivalent to stating:

$$
2 E\left\{U_{r}\right\} E\left\{U_{q}^{-1}\right\} \geq E\left\{V_{r}^{-1}\right\} E\left\{V_{q}\right\}
$$

holds uniformly in $\left(\sigma_{\bar{x}_{1}}^{2}, \sigma_{\bar{x}_{2}}^{2}, \ldots, \sigma_{\bar{x}_{p}}^{2}\right)$.

Proof. According to Lemma 2.4, the probability $P\left\{\left|\hat{\mu}_{G D \mid p}-\mu\right| \leq\left|\hat{\mu}_{G D \mid q}-\mu\right|\right\}$ can be denoted as:

$$
\begin{aligned}
\gamma & =\gamma\left(\sigma_{\bar{x}_{1}}^{2}, \sigma_{\bar{x}_{2}}^{2}, \ldots, \sigma_{\bar{x}_{p}}^{2}\right) \\
& =1 / \pi\left(E\left\{\arctan \left(U_{r} U_{q}^{-1}\right)\right\}+E\left\{\arctan \left(U_{r} U_{q}^{-1}+2 V_{r} V_{q}^{-1}\right)\right\}\right) .
\end{aligned}
$$


The expectations are taken with respect to the independent random variables $Y_{i}$ 's.

Let $\sigma_{\bar{x}_{i}}^{-2}=\sigma_{\bar{x}_{1}}^{-2} \tau_{i}$, for $i=1,2, \ldots, q$; and $\sigma_{\bar{x}_{i}}^{-2}=\sigma_{\bar{x}_{1}}^{2} \tau_{i}$, for $i=q+1, q+2, \ldots, q+r$. Here $\tau_{i}$ 's are in the range of $[0, \infty)$, except $\tau_{1}=1$. ${ }^{a}$ So $U_{q}=\sigma_{\bar{x}_{1}}^{-1} U_{q}^{*}, V_{q}=\sigma_{\bar{x}_{1}} V_{q}^{*}, U_{r}=\sigma_{\bar{x}_{1}} U_{r}^{*}$, and $V_{r}=\sigma_{\bar{x}_{1}}^{-1} V_{r}^{*}$, where

$$
\begin{aligned}
& U_{q}^{*}=\left(\sum_{i=1}^{q} \tau_{i} Y_{i}^{2}\right)^{-1 / 2}\left(\sum_{i=1}^{q} \tau_{i} Y_{i}\right), \quad V_{q}^{*}=\left(\sum_{i=1}^{q} \tau_{i} Y_{i}^{2}\right)^{-1 / 2}, \\
& U_{r}^{*}=\left(\sum_{i=q+1}^{q+r} \tau_{i} Y_{i}^{2}\right)^{-1 / 2}\left(\sum_{i=q+1}^{q+r} \tau_{i} Y_{i}\right), V_{r}^{*}=\left(\sum_{i=q+1}^{q+r} \tau_{i} Y_{i}^{2}\right)^{-1 / 2} .
\end{aligned}
$$

The probability in equation (3.2) will be

$$
\begin{aligned}
\gamma= & \gamma\left(\sigma_{\bar{x}_{1}}^{2}, \tau_{1}, \tau_{2}, \ldots, \tau_{p}\right) \\
= & 1 / \pi\left(E\left\{\arctan \left(\sigma_{\bar{x}_{1}}^{2} U_{r}^{*}\left(U_{q}^{*}\right)^{-1}\right)\right\}\right. \\
& \left.+E\left\{\arctan \left(\sigma_{\bar{x}_{1}}^{2} U_{r}^{*}\left(U_{q}^{*}\right)^{-1}+2{\sigma_{\bar{x}}}_{\bar{x}_{1}}^{-2} V_{r}^{*}\left(V_{q}^{*}\right)^{-1}\right)\right\}\right) .
\end{aligned}
$$

The dominated converge theorem implies that:

$$
\gamma\left(\sigma_{\bar{x}_{1}}^{2}\right) \rightarrow 1 / \pi(E\{\arctan 0\}+E\{\arctan \infty\})=1 / 2 \text { as } \sigma_{\bar{x}_{1}}^{2} \rightarrow 0 .
$$

Next, we will show that $\gamma \geq 1 / 2$ uniformly in $\left(\sigma_{\bar{x}_{1}}^{2}, \sigma_{\bar{x}_{2}}^{2}, \ldots, \sigma_{\bar{x}_{p}}^{2}\right)$, only if $2 E\left\{U_{r}\right\} E\left\{U_{q}^{-1}\right\} \geq$ $E\left\{V_{q}\right\} E\left\{V_{r}^{-1}\right\}$ holds uniformly in $\left(\sigma_{\bar{x}_{1}}^{2}, \sigma_{\bar{x}_{2}}^{2}, \ldots, \sigma_{\bar{x}_{p}}^{2}\right)$.

The derivative of $\gamma$ with respect to $\sigma_{\bar{x}_{1}}^{2}$, is given by

$$
\begin{aligned}
& \frac{\partial}{\partial \sigma_{\bar{x}_{1}}^{2}} \pi \gamma \\
& =E\left\{\frac{U_{r}^{*}\left(U_{q}^{*}\right)^{-1}-2 \sigma_{\bar{x}_{1}}^{-4} V_{r}^{*}\left(V_{q}^{*}\right)^{-1}}{1+\left(\sigma_{\bar{x}_{1}}^{2} U_{r}^{*}\left(U_{q}^{*}\right)^{-1}+2 \sigma_{\bar{x}_{1}}^{-2} V_{r}^{*}\left(V_{q}^{*}\right)^{-1}\right)^{2}}\right\}+E\left\{\frac{U_{r}^{*}\left(U_{q}^{*}\right)^{-1}}{1+\sigma_{\bar{x}_{1}}^{4} U_{r}^{* 2}\left(U_{q}^{*}\right)^{-2}}\right\} \\
& =E\left\{\frac{U_{r}^{*}\left(U_{q}^{*}\right)^{-1} \sigma_{\bar{x}_{1}}^{4}-2 V_{r}^{*}\left(V_{q}^{*}\right)^{-1}}{\sigma_{\bar{x}_{1}}^{4}\left(1+\sigma_{\bar{x}_{1}}^{4} U_{r}^{* 2}\left(U_{q}^{*}\right)^{-2}\right)+4 V_{r}^{*}\left(V_{q}^{*}\right)^{-1}\left(\sigma_{\bar{x}_{1}}^{4} U_{r}^{*}\left(U_{q}^{*}\right)^{-1}+V_{r}^{*}\left(V_{q}^{*}\right)^{-1}\right)}\right\} \\
& +E\left\{\frac{U_{r}^{*}\left(U_{q}^{*}\right)^{-1}}{1+\sigma_{\bar{x}_{1}}^{4} U_{r}^{* 2}\left(U_{q}^{*}\right)^{-2}}\right\} .
\end{aligned}
$$

To check whether the inequality:

$$
\left.E\left\{-\frac{1}{2} V_{q}^{*}\left(V_{r}^{*}\right)^{-1}+U_{r}^{*}\left(U_{q}^{*}\right)^{-1}\right)\right\} \geq 0
$$

is a necessary condition, we assume that inequality (3.4) is not true for some $\left(\tau_{2}, \ldots, \tau_{p}\right)$. Then we notice that

$$
\left.\left.\frac{\partial}{\partial \sigma_{\bar{x}_{1}}^{2}} \gamma\right|_{\sigma_{\bar{x}_{1}}^{2}=0}=1 / \pi E\left\{-\frac{1}{2} V_{q}^{*}\left(V_{r}^{*}\right)^{-1}+U_{r}^{*}\left(U_{q}^{*}\right)^{-1}\right)\right\}<0,
$$

which leads to $\gamma$ being non-decreasing and contradicts that $\gamma \geq 1 / 2$ for $\sigma_{\bar{x}_{1}}^{2}$ in a small region near 0 .

an inequality (3.4), we are going to prove, $2 E\left\{U_{r}\right\} E\left\{U_{q}^{-1}\right\} \geq E\left\{V_{q}\right\} E\left\{V_{r}^{-1}\right\}$, it will follow that $U_{\star}$ 's and $V_{\star}^{-1}$ 's are proportional to $\sigma_{\star}^{-2}$. 
It is easy to verify that inequality (3.4) is equivalent to inequality (3.1).

\section{When is combining preferable ? Several Sufficient Conditions}

Theorem 4.1. If

$$
E\left\{\frac{-2^{-1}}{\sigma_{\bar{x}_{1}}^{4} U_{r}^{*}\left(U_{q}^{*}\right)^{-1}+V_{r}^{*}\left(V_{q}^{*}\right)^{-1}}\right\}+E\left\{\frac{U_{r}^{*}\left(U_{q}^{*}\right)^{-1}}{1+\sigma_{\bar{x}_{1}}^{4} U_{r}^{* 2}\left(U_{q}^{*}\right)^{-2}}\right\} \geq 0,
$$

then $P\left\{\left|\hat{\mu}_{G D \mid p}-\mu\right| \leq\left|\hat{\mu}_{G D \mid q}-\mu\right|\right\} \geq 1 / 2$ holds uniformly in $\left(\mu, \sigma_{\bar{x}_{1}}^{2}, \sigma_{\bar{x}_{2}}^{2}, \ldots, \sigma_{\bar{x}_{p}}^{2}\right)$.

Proof. Continue from equation (3.3) in Theorem 3.1, we obtain the following:

$$
\begin{aligned}
& \frac{\partial}{\partial \sigma_{\bar{x}_{1}}^{2}} \pi \gamma \\
& =E\left\{\frac{U_{r}^{*}\left(U_{q}^{*}\right)^{-1} \sigma_{\bar{x}_{1}}^{4}-2 V_{r}^{*}\left(V_{q}^{*}\right)^{-1}}{\sigma_{\bar{x}_{1}}^{4}\left(1+\sigma_{\bar{x}_{1}}^{4} U_{r}^{* 2}\left(U_{q}^{*}\right)^{-2}\right)+4 V_{r}^{*}\left(V_{q}^{*}\right)^{-1}\left(\sigma_{\bar{x}_{1}}^{4} U_{r}^{*}\left(U_{q}^{*}\right)^{-1}+V_{r}^{*}\left(V_{q}^{*}\right)^{-1}\right)}\right\} \\
& +E\left\{\frac{U_{r}^{*}\left(U_{q}^{*}\right)^{-1}}{1+\sigma_{\bar{x}_{1}}^{4} U_{r}^{* 2}\left(U_{q}^{*}\right)^{-2}}\right\} \\
& \geq E\left\{\frac{-2 V_{r}^{*}\left(V_{q}^{*}\right)^{-1}}{\sigma_{\bar{x}_{1}}^{4}\left(1+\sigma_{\bar{x}_{1}}^{4} U_{r}^{* 2}\left(U_{q}^{*}\right)^{-2}\right)+4 V_{r}^{*}\left(V_{q}^{*}\right)^{-1}\left(\sigma_{\bar{x}_{1}}^{4} U_{r}^{*}\left(U_{q}^{*}\right)^{-1}+V_{r}^{*}\left(V_{q}^{*}\right)^{-1}\right)}\right\} \\
& +E\left\{\frac{U_{r}^{*}\left(U_{q}^{*}\right)^{-1}}{1+\sigma_{\bar{x}_{1}}^{4} U_{r}^{* 2}\left(U_{q}^{*}\right)^{-2}}\right\} \\
& \geq E\left\{\frac{-2 V_{r}^{*}\left(V_{q}^{*}\right)^{-1}}{4 V_{r}^{*}\left(V_{q}^{*}\right)^{-1}\left(\sigma_{\bar{x}_{1}}^{4} U_{r}^{*}\left(U_{q}^{*}\right)^{-1}+V_{r}^{*}\left(V_{q}^{*}\right)^{-1}\right)}\right\}+E\left\{\frac{U_{r}^{*}\left(U_{q}^{*}\right)^{-1}}{1+\sigma_{\bar{x}_{1}}^{4} U_{r}^{* 2}\left(U_{q}^{*}\right)^{-2}}\right\} \\
& =E\left\{\frac{-2^{-1}}{\sigma_{\bar{x}_{1}}^{4} U_{r}^{*}\left(U_{q}^{*}\right)^{-1}+V_{r}^{*}\left(V_{q}^{*}\right)^{-1}}\right\}+E\left\{\frac{U_{r}^{*}\left(U_{q}^{*}\right)^{-1}}{1+\sigma_{\bar{x}_{1}}^{4} U_{r}^{* 2}\left(U_{q}^{*}\right)^{-2}}\right\} .
\end{aligned}
$$

Hence if

$$
E\left\{\frac{-2^{-1}}{\sigma_{\bar{x}_{1}}^{4} U_{r}^{*}\left(U_{q}^{*}\right)^{-1}+V_{r}^{*}\left(V_{q}^{*}\right)^{-1}}\right\}+E\left\{\frac{U_{r}^{*}\left(U_{q}^{*}\right)^{-1}}{1+\sigma_{\bar{x}_{1}}^{4} U_{r}^{* 2}\left(U_{q}^{*}\right)^{-2}}\right\} \geq 0,
$$

then $\frac{\partial}{\partial \sigma_{\bar{x}_{1}}^{2}} \pi \gamma \geq 0$, which leads to $\gamma\left(\sigma_{\bar{x}_{1}}^{2}, \tau_{2}, \ldots, \tau_{p}\right)$ being non-decreasing in $\sigma_{\bar{x}_{1}}^{2}$ for any of $\left(\tau_{2}, \ldots, \tau_{p}\right)$. The dominated converge theorem implies that $\gamma\left(\sigma_{\bar{x}_{1}}^{2}\right) \rightarrow 1 / \pi(E\{\arctan 0\}+E\{\arctan \infty\})=1 / 2$ as $\sigma_{\bar{x}_{1}}^{2} \rightarrow 0$. For any other finite $\left(\tau_{2}, \ldots, \tau_{p}\right)$ and $\sigma_{\bar{x}_{1}}^{2}$, we have $\gamma\left(\sigma_{\bar{x}_{1}}^{2}, \tau_{2}, \ldots, \tau_{p}\right) \geq \gamma\left(0, \tau_{2}, \ldots, \tau_{p}\right)=1 / 2$. At the boundary where one or some $\tau_{i}$ 's go to $\infty$, we have $\gamma\left(\sigma_{\bar{x}_{1}}^{2}, \tau_{2}, \ldots, \tau_{p}\right)=1$ or $1 / 2>1 / 2$. Hence $\gamma \geq 1 / 2$ holds uniformly in $\left(\sigma_{\bar{x}_{1}}^{2}, \sigma_{\bar{x}_{2}}^{2}, \ldots, \sigma_{\bar{x}_{p}}^{2}\right)$.

Based on Theorem 4.1, we derive several sufficient conditions in the sequel for $P\left\{\left|\hat{\mu}_{G D \mid p}-\mu\right| \leq\right.$ $\left.\left|\hat{\mu}_{G D \mid q}-\mu\right|\right\} \geq 1 / 2$ to hold uniformly in $\left(\mu, \sigma_{\bar{x}_{1}}^{2}, \sigma_{\bar{x}_{2}}^{2}, \ldots, \sigma_{\bar{x}_{p}}^{2}\right)$. 


\section{Corollary 4.2.}

$$
2 E\left(U_{r}\right) E\left(U_{q}^{-1}\right) \geq \frac{E\left(U_{q}\right)}{\min _{i=1, \ldots, q}\left(\sigma_{\bar{x}_{i}}^{-2}\right)} E\left(V_{r}^{-1}\right)
$$

is a sufficient condition for $P\left\{\left|\hat{\mu}_{G D \mid p}-\mu\right| \leq\left|\hat{\mu}_{G D \mid q}-\mu\right|\right\} \geq 1 / 2$ to hold uniformly in $\left(\mu, \sigma_{\bar{x}_{1}}^{2}, \sigma_{\bar{x}_{2}}^{2}, \ldots, \sigma_{\bar{x}_{p}}^{2}\right)$.

Proof. It is easy to state that

$$
2 E\left(U_{r}\right) E\left(U_{q}^{-1}\right) \geq \frac{E\left(U_{q}\right)}{\min _{i=1, \ldots, q}\left(\sigma_{\bar{x}_{i}}^{-2}\right)} E\left(V_{r}^{-1}\right)
$$

is equivalent to

$$
2 E\left(U_{r}^{*}\right) E\left(U_{q}^{*-1}\right) \geq \frac{E\left(U_{q}^{*}\right)}{\min _{i=1, \ldots, q}\left(\tau_{i}\right)} E\left(V_{r}^{*-1}\right) .
$$

From Theorem 4.1, we only need to show the validity of inequality (4.1).

At the left side of inequality (4.1), we have the following inequality for the second term:

$$
E\left\{\frac{\left.U_{r}^{*}\left(U_{q}^{*}\right)^{-1}\right)}{1+\sigma_{\bar{x}_{1}}^{4} U_{r}^{* 2}\left(U_{q}^{*}\right)^{-2}}\right\} \geq E\left\{\frac{\left.U_{r}^{*}\left(U_{q}^{*}\right)^{-1}\right)}{1+\sigma_{\bar{x}_{1}}^{4}\left(\sum_{i=q+1}^{q+r} \tau_{i}\right)\left(\min _{i=1, \ldots, q}\left(\tau_{i}\right)\right)^{-1}}\right\} .
$$

This is because of $U_{r}^{* 2} \leq \sum_{i=q+1}^{q+r} \tau_{i}$ and $\left(U_{q}^{*}\right)^{-2} \leq\left(\min _{i=1, \ldots, q}\left(\tau_{i}\right)\right)^{-1}$ following by Lemma 2.1.

We also have:

$$
\begin{aligned}
& \left(1+\sigma_{\bar{x}_{1}}^{4}\left(\sum_{i=q+1}^{q+r} \tau_{i}\right)\left(\min _{i=1, \ldots, q}\left(\tau_{i}\right)\right)^{-1}\right) E\left\{\left\{\sigma_{\bar{x}_{1}}^{4} U_{r}^{*}\left(U_{q}^{*}\right)^{-1}+V_{r}^{*}\left(V_{q}^{*}\right)^{-1}\right\}^{-1}\right\} \\
\leq & \left(1+\frac{\sigma_{\bar{x}_{1}}^{4}\left(\sum_{i=q+1}^{q+r} \tau_{i}\right)}{\min _{i=1, \ldots, q}\left(\tau_{i}\right)}\right)^{-1} E\left\{\frac{\sigma_{\bar{x}_{1}}^{4}\left(\sum_{i=q+1}^{q+r} \tau_{i}\right)^{2}}{\left(\min _{i=1, \ldots, q}\left(\tau_{i}\right)\right)^{2}}\left(U_{r}^{*}\right)^{-1}\left(U_{q}^{*}\right)+V_{q}^{*}\left(V_{r}^{*}\right)^{-1}\right\} \\
\leq & \max \left\{\frac{E\left(U_{q}^{*}\right)}{\min _{i=1, \ldots, q}\left(\tau_{i}\right)}\left(\sum_{i=q+1}^{q+r} \tau_{i}\right) E\left(U_{r}^{*-1}\right), E\left(V_{q}^{*}\right) E\left(V_{r}^{*-1}\right)\right\} .
\end{aligned}
$$

The inequality (4.3) follows from Jensen's inequality:

$$
(1+a) E(a A+B)^{-1} \leq(1+a)^{-1} E\left(a A^{-1}+B^{-1}\right),
$$

where $a=\frac{\sigma_{\bar{x}_{1}}^{4}\left(\sum_{i=q+1}^{q+r} \tau_{i}\right)}{\min _{i=1, \ldots, q}\left(\tau_{i}\right)}, A=\frac{\min _{i=1, \ldots, q}\left(\tau_{i}\right)}{\left(\sum_{i=q+1}^{q+r} \tau_{i}\right)} U_{r}^{*}\left(U_{q}^{*}\right)^{-1}$, and $B=V_{r}^{*}\left(V_{q}^{*}\right)^{-1}$.

From Lemma 2.3, we know

$$
\left(\sum_{i=q+1}^{q+r} \tau_{i}\right) E\left(U_{r}^{*-1}\right) \leq E\left(V_{r}^{*-1}\right)
$$

From Lemma 2.2 and the fact that $\frac{U_{q}^{*}}{\min _{i=1, \ldots, q}\left(\tau_{i}\right)} \geq U_{q}^{*-1}$, we note

$$
\frac{E\left(U_{q}^{*}\right)}{\min _{i=1, \ldots, q}\left(\tau_{i}\right)} \geq E\left(U_{q}^{*-1}\right) \geq E\left(V_{q}^{*}\right)
$$


So we have the following :

$$
\begin{aligned}
& \max \left\{\frac{E\left(U_{q}^{*}\right)}{\min _{i=1, \ldots, q}\left(\tau_{i}\right)}\left(\sum_{i=q+1}^{q+r} \tau_{i}\right) E\left(U_{r}^{*-1}\right), E\left(V_{q}^{*}\right) E\left(V_{r}^{*-1}\right)\right\} \\
& \leq \frac{E\left(U_{q}^{*}\right)}{\min _{i=1, \ldots, q}\left(\tau_{i}\right)} E\left(V_{r}^{*-1}\right) .
\end{aligned}
$$

Then at the left side of inequality (4.1), we have the following inequality for the first term:

$$
\begin{aligned}
& E\left\{\left\{\sigma_{\bar{x}_{1}}^{4} U_{r}^{*}\left(U_{q}^{*}\right)^{-1}+V_{r}^{*}\left(V_{q}^{*}\right)^{-1}\right\}^{-1}\right\} \leq\left(1+\frac{\sigma_{\bar{x}_{1}}^{4}\left(\sum_{i=q+1}^{q+r} \tau_{i}\right)}{\min _{i=1, \ldots, q}\left(\tau_{i}\right)}\right)^{-1} \\
& \max \left\{\frac{E\left(U_{q}^{*}\right)}{\min _{i=1, \ldots, q}\left(\tau_{i}\right)}\left(\sum_{i=q+1}^{q+r} \tau_{i}\right) E\left(U_{r}^{*-1}\right), E\left(V_{q}^{*}\right) E\left(V_{r}^{*-1}\right)\right\} \\
& \leq\left(1+\frac{\sigma_{\bar{x}_{1}}^{4}\left(\sum_{i=q+1}^{q+r} \tau_{i}\right)}{\min _{i=1, \ldots, q}\left(\tau_{i}\right)}\right)^{-1} \frac{E\left(U_{q}^{*}\right)}{\min _{i=1, \ldots, q}\left(\tau_{i}\right)} E\left(V_{r}^{*-1}\right)
\end{aligned}
$$

Combining the above and inequality (4.2), we have

$$
\begin{aligned}
& \frac{\partial}{\partial \sigma_{\bar{x}_{1}}^{2}} \pi \gamma \geq E\left\{\frac{-2^{-1}}{\sigma_{\bar{x}_{1}}^{4} U_{r}^{*}\left(U_{q}^{*}\right)^{-1}+V_{r}^{*}\left(V_{q}^{*}\right)^{-1}}\right\}+E\left\{\frac{\left.U_{r}^{*}\left(U_{q}^{*}\right)^{-1}\right)}{1+\sigma_{\bar{x}_{1}}^{4} U_{r}^{* 2}\left(U_{q}^{*}\right)^{-2}}\right\} \\
\geq & \left\{-2^{-1} \frac{E\left(U_{q}^{*}\right)}{\min _{i=1, \ldots, q}\left(\tau_{i}\right)} E\left(V_{r}^{*-1}\right)+E\left\{U_{r}^{*}\left(U_{q}^{*}\right)^{-1}\right\}\right\}\left(1+\frac{\sigma_{\bar{x}_{1}}^{4}\left(\sum_{i=q+1}^{q+r} \tau_{i}\right)}{\min _{i=1, \ldots, q}\left(\tau_{i}\right)}\right)^{-1} .
\end{aligned}
$$

If

$$
-2^{-1} \frac{E\left(U_{q}^{*}\right)}{\min _{i=1, \ldots, q}\left(\tau_{i}\right)} E\left(V_{r}^{*-1}\right)+E\left\{U_{r}^{*}\left(U_{q}^{*}\right)^{-1}\right\} \geq 0
$$

then

$$
\frac{\partial}{\partial \sigma_{\bar{x}_{1}}^{2}} \pi \gamma \geq 0
$$

Therefore a sufficient condition for $P\left\{\left|\hat{\mu}_{G D \mid p}-\mu\right| \leq\left|\hat{\mu}_{G D \mid q}-\mu\right|\right\} \geq 1 / 2$ to hold uniformly in $\left(\mu, \sigma_{\bar{x}_{1}}^{2}, \sigma_{\bar{x}_{2}}^{2}, \ldots, \sigma_{\bar{x}_{p}}^{2}\right)$ is:

$$
2 E\left(U_{r}\right) E\left(U_{q}^{-1}\right) \geq \frac{E\left(U_{q}\right)}{\min _{i=1, \ldots, q}\left(\sigma_{\bar{x}_{i}}^{-2}\right)} E\left(V_{r}^{-1}\right) .
$$

Another corollary is given in the following:

\section{Corollary 4.3.}

$$
2 E\left(U_{r}\right) E\left(U_{q}\right) \geq \sqrt{\sum_{i=1}^{q} \sigma_{\bar{x}_{i}}^{-2}} E\left(V_{r}^{-1}\right),
$$


or

$$
2 E\left(U_{r}\right) E\left(U_{q}\right) \geq E\left(V_{q}^{-1}\right) E\left(V_{r}^{-1}\right) .
$$

is a sufficient condition for $P\left\{\left|\hat{\mu}_{G D \mid p}-\mu\right| \leq\left|\hat{\mu}_{G D \mid q}-\mu\right|\right\} \geq 1 / 2$ to hold uniformly in $\left(\mu, \sigma_{\bar{x}_{1}}^{2}, \sigma_{\bar{x}_{2}}^{2}, \ldots, \sigma_{\bar{x}_{p}}^{2}\right)$.

Proof. We need to validate inequality (4.1) in Theorem 4.1. On the left side of inequality (4.1), we have the following inequality for the second term:

$$
E\left\{\frac{\left.U_{r}^{*}\left(U_{q}^{*}\right)^{-1}\right)}{1+\sigma_{\bar{x}_{1}}^{4} U_{r}^{* 2}\left(U_{q}^{*}\right)^{-2}}\right\} \geq E\left\{\frac{U_{r}^{*} U_{q}^{*}}{\sum_{i=1}^{q} \tau_{i}+\sigma_{\bar{x}_{1}}^{4}\left(\sum_{i=q+1}^{q+r} \tau_{i}\right)}\right\} .
$$

It is because of the facts $U_{r}^{* 2} \leq \sum_{i=q+1}^{q+r} \tau_{i}$ and $U_{q}^{* 2} \leq \sum_{i=1}^{q} \tau_{i}$ following from lemma 2.1.

We also have:

$$
\begin{aligned}
& \left(\sum_{i=1}^{q} \tau_{i}+\sigma_{\bar{x}_{1}}^{4}\left(\sum_{i=q+1}^{q+r} \tau_{i}\right)\right) E\left\{\left\{\sigma_{\bar{x}_{1}}^{4} U_{r}^{*}\left(U_{q}^{*}\right)^{-1}+V_{r}^{*}\left(V_{q}^{*}\right)^{-1}\right\}^{-1}\right\} \\
\leq & \left(\sum_{i=1}^{q} \tau_{i}+\sigma_{\bar{x}_{1}}^{4}\left(\sum_{i=q+1}^{q+r} \tau_{i}\right)\right)^{-1} E\left\{\sigma_{\bar{x}_{1}}^{4}\left(\sum_{i=q+1}^{q+r} \tau_{i}\right)^{2}\left(U_{r}^{*}\right)^{-1}\left(U_{q}^{*}\right)+\left(\sum_{i=1}^{q} \tau_{i}\right)^{2} V_{q}^{*}\left(V_{r}^{*}\right)^{-1}\right\} \\
\leq & \max \left\{\left(\sum_{i=q+1}^{q+r} \tau_{i}\right) E\left(U_{r}^{*}\right)^{-1} E\left(U_{q}^{*}\right),\left(\sum_{i=1}^{q} \tau_{i}\right) E\left(V_{q}^{*}\right) E\left(V_{r}^{*}\right)^{-1}\right\} .
\end{aligned}
$$

The inequality (4.5) is from Jensen's inequality.

So at the left side of inequality (4.1), we have the following inequality for the first term:

$$
\begin{array}{r}
E\left\{\left\{\sigma_{\bar{x}_{1}}^{4} U_{r}^{*}\left(U_{q}^{*}\right)^{-1}+V_{r}^{*}\left(V_{q}^{*}\right)^{-1}\right\}^{-1}\right\} \leq\left(\sum_{i=1}^{q} \tau_{i}+\sigma_{\bar{x}_{1}}^{4}\left(\sum_{i=q+1}^{q+r} \tau_{i}\right)\right)^{-1} \\
\max \left\{\left(\sum_{i=q+1}^{q+r} \tau_{i}\right) E\left(U_{r}^{*}\right)^{-1} E\left(U_{q}^{*}\right),\left(\sum_{i=1}^{q} \tau_{i}\right) E\left(V_{q}^{*}\right) E\left(V_{r}^{*}\right)^{-1}\right\} .
\end{array}
$$

From Lemma 2.3 we have:

$$
\left(\sum_{i=q+1}^{q+r} \tau_{i}\right) E\left(U_{r}^{*-1}\right) \leq E\left(V_{r}^{*-1}\right)
$$

The upper bound of $E\left(U_{q}^{*}\right)$ and $\left(\sum_{i=1}^{q} \tau_{i}\right) E\left(V_{q}^{*}\right)$ is $\sqrt{\sum_{i=1}^{q} \tau_{i}}$. This is due to Lemma 2.1 and Lemma 2.2, respectively.

Another upper bound for $\left(\sum_{i=1}^{q} \tau_{i}\right) E\left(V_{q}^{*}\right)$ is $E\left(V_{q}^{*-1}\right)$. Since

$$
\left(\sum_{i=1}^{q} \tau_{i}\right) E\left(V_{q}^{*}\right)=\frac{\left(\sum_{i=1}^{q} \tau_{i}\right) E\left(U_{q}^{*-1}\right) E\left(V_{q}^{*}\right)}{E\left(U_{q}^{*-1}\right)} \leq \frac{E\left(V_{q}^{*-1}\right) E\left(V_{q}^{*}\right)}{E\left(U_{q}^{*-1}\right)} \leq E\left(V_{q}^{*-1}\right),
$$


then we have

$$
\max \left\{\left(\sum_{i=q+1}^{q+r} \tau_{i}\right) E\left(U_{r}^{*}\right)^{-1} E\left(U_{q}^{*}\right),\left(\sum_{i=1}^{q} \tau_{i}\right) E\left(V_{q}^{*}\right) E\left(V_{r}^{*}\right)^{-1}\right\} \leq \sqrt{\sum_{i=1}^{q} \tau_{i} E\left(V_{r}^{*-1}\right),}
$$

or

$$
\begin{aligned}
\max & \left\{\left(\sum_{i=q+1}^{q+r} \tau_{i}\right) E\left(U_{r}^{*}\right)^{-1} E\left(U_{q}^{*}\right),\left(\sum_{i=1}^{q} \tau_{i}\right) E\left(V_{q}^{*}\right) E\left(V_{r}^{*}\right)^{-1}\right\} \leq \\
& \max \left\{E\left(V_{q}^{*-1}\right), E\left(U_{q}^{*}\right)\right\} E\left(V_{r}^{*-1}\right)=E\left(V_{q}^{*-1}\right) E\left(V_{r}^{*-1}\right) .
\end{aligned}
$$

Combining inequalities (4.4), (4.6) and (4.7), we have the following:

$$
\begin{aligned}
& \frac{\partial}{\partial \sigma_{\bar{x}_{1}}^{2}} \pi \gamma \geq E\left\{\frac{-2^{-1}}{\sigma_{\bar{x}_{1}}^{4} U_{r}^{*}\left(U_{q}^{*}\right)^{-1}+V_{r}^{*}\left(V_{q}^{*}\right)^{-1}}\right\}+E\left\{\frac{\left.U_{r}^{*}\left(U_{q}^{*}\right)^{-1}\right)}{1+\sigma_{\bar{x}_{1}}^{4} U_{r}^{* 2}\left(U_{q}^{*}\right)^{-2}}\right\} \\
& \geq\left\{-2^{-1} \sqrt{\left.\sum_{i=1}^{q} \tau_{i} E\left(V_{r}^{*-1}\right)+E\left(U_{r}^{*}\right) E\left(U_{q}^{*}\right)\right\}\left(\sum_{i=1}^{q} \tau_{i}+\sigma_{\bar{x}_{1}}^{4}\left(\sum_{i=q+1}^{q+r} \tau_{i}\right)\right)^{-1},}\right.
\end{aligned}
$$

while combining inequalities (4.4), (4.6) and (4.8), we obtain

$$
\begin{aligned}
& \frac{\partial}{\partial \sigma_{\bar{x}_{1}}^{2}} \pi \gamma \geq E\left\{\frac{-2^{-1}}{\sigma_{\bar{x}_{1}}^{4} U_{r}^{*}\left(U_{q}^{*}\right)^{-1}+V_{r}^{*}\left(V_{q}^{*}\right)^{-1}}\right\}+E\left\{\frac{\left.U_{r}^{*}\left(U_{q}^{*}\right)^{-1}\right)}{1+\sigma_{\bar{x}_{1}}^{4} U_{r}^{* 2}\left(U_{q}^{*}\right)^{-2}}\right\} \\
\geq & \left\{-2^{-1} E\left(V_{q}^{*-1}\right) E\left(V_{r}^{*-1}\right)+E\left(U_{r}^{*}\right) E\left(U_{q}^{*}\right)\right\}\left(\sum_{i=1}^{q} \tau_{i}+\sigma_{\bar{x}_{1}}^{4}\left(\sum_{i=q+1}^{q+r} \tau_{i}\right)\right)^{-1} .
\end{aligned}
$$

So if

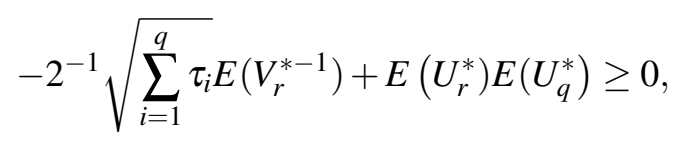

or

$$
-2^{-1} E\left(V_{q}^{*-1}\right) E\left(V_{r}^{*-1}\right)+E\left(U_{r}^{*}\right) E\left(U_{q}^{*}\right) \geq 0
$$

then

$$
\frac{\partial}{\partial \sigma_{\bar{x}_{1}}^{2}} \pi \gamma \geq 0
$$

Hence it is equivalent to saying that a sufficient condition for $P\left\{\left|\hat{\mu}_{G D \mid p}-\mu\right| \leq\left|\hat{\mu}_{G D \mid q}-\mu\right|\right\} \geq 1 / 2$ to hold uniformly in $\left(\mu, \sigma_{\bar{x}_{1}}^{2}, \sigma_{\bar{x}_{2}}^{2}, \ldots, \sigma_{\bar{x}_{p}}^{2}\right)$ is:

$$
2 E\left(U_{r}\right) E\left(U_{q}\right) \geq \sqrt{\sum_{i=1}^{q} \sigma_{\bar{x}_{i}}^{-2}} E\left(V_{r}^{-1}\right)
$$

or

$$
2 E\left(U_{r}\right) E\left(U_{q}\right) \geq E\left(V_{q}^{-1}\right) E\left(V_{r}^{-1}\right) .
$$




\section{Sample Size Discussion}

In this section we will discuss the sample size requirement to ensure the sufficient condition provided in Corollary 4.3:

$$
2 E\left(U_{r}\right) E\left(U_{q}\right) \geq E\left(V_{q}^{-1}\right) E\left(V_{r}^{-1}\right)
$$

Which is the same as:

$$
\begin{aligned}
& 2 E\left\{\left(\sum_{i=1}^{q} \theta_{i} Y_{i}\right)\left(\sum_{i=1}^{q} \theta_{i} Y_{i}^{2}\right)^{-\frac{1}{2}}\right\} E\left\{\left(\sum_{i=q+1}^{q+r} \theta_{i} Y_{i}\right)\left(\sum_{i=q+1}^{q+r} \theta_{i} Y_{i}^{2}\right)^{-\frac{1}{2}}\right\} \\
& \geq E\left(\sum_{i=1}^{q} \theta_{i} Y_{i}^{2}\right)^{\frac{1}{2}} E\left(\sum_{i=q+1}^{q+r} \theta_{i} Y_{i}^{2}\right)^{\frac{1}{2}},
\end{aligned}
$$

$\theta_{i}=\sigma_{\bar{x}_{i}}^{-2}, i=1,2, \ldots, p$. We specified the following theorem:

Theorem 5.1. Let $Y_{1}, \ldots, Y_{p}$ be independent random variables such that $Y_{i} \sim \frac{m_{i}}{\chi^{2}\left(m_{i}\right)}$, for $i=$ $1,2, \ldots, p$, and let $p=q+r$. Then for any $\theta_{i}>0(i=1,2, \ldots, p)$,

$$
\begin{aligned}
& 2 E\left\{\left(\sum_{i=1}^{q} \theta_{i} Y_{i}\right)\left(\sum_{i=1}^{q} \theta_{i} Y_{i}^{2}\right)^{-\frac{1}{2}}\right\} E\left\{\left(\sum_{i=q+1}^{q+r} \theta_{i} Y_{i}\right)\left(\sum_{i=q+1}^{q+r} \theta_{i} Y_{i}^{2}\right)^{-\frac{1}{2}}\right\} \\
& \geq E\left(\sum_{i=1}^{q} \theta_{i} Y_{i}^{2}\right)^{\frac{1}{2}} E\left(\sum_{i=q+1}^{q+r} \theta_{i} Y_{i}^{2}\right)^{\frac{1}{2}}
\end{aligned}
$$

if there exists $1<b<2$, such that

$$
m_{i}^{-1}\left(m_{i}-4\right)+2\left(\sum_{j=1}^{q} m_{j}\right)^{-1} \geq \frac{1}{b}
$$

for all $i=1,2, \ldots, q$, and

$$
m_{i}^{-1}\left(m_{i}-4\right)+2\left(\sum_{j=q+1}^{q+r} m_{j}\right)^{-1} \geq \frac{b}{2}
$$

for all $i=q+1, q+2, \ldots, q+r=p$.

Proof. Define:

$$
g_{1}\left(Y_{i}\right)=E\left\{Y_{i}\left(\sum_{j=1}^{q} \theta_{j} Y_{j}^{2}\right)^{-\frac{1}{2}} \mid Y_{i}\right\},
$$

for $i=1,2, \ldots, q$; and define:

$$
g_{2}\left(Y_{i}\right)=E\left\{Y_{i}\left(\sum_{j=q+1}^{q+r} \theta_{j} Y_{j}^{2}\right)^{-\frac{1}{2}} \mid Y_{i}\right\},
$$

for $i=q+1, q+2, \ldots, q+r=p$.

From [7] and [2], we know the following for $i=1,2, \ldots, q$ :

$$
m_{i} E\left(g_{1}\left(Y_{i}\right)\right)=\left(m_{i}-2\right) E\left(Y_{i} g_{1}\left(Y_{i}\right)\right)-2 E\left(Y_{i}^{2} g^{\prime}{ }_{1}\left(Y_{i}\right)\right),
$$


and similarly for $i=q+1, q+2, \ldots, q+r=p$, we have:

$$
m_{i} E\left(g_{2}\left(Y_{i}\right)\right)=\left(m_{i}-2\right) E\left(Y_{i} g_{2}\left(Y_{i}\right)\right)-2 E\left(Y_{i}^{2} g_{2}^{\prime}\left(Y_{i}\right)\right) .
$$

Then,

$$
g^{\prime}{ }_{1}\left(Y_{i}\right)=E\left\{\left(\sum_{j=1}^{q} \theta_{j} Y_{j}^{2}\right)^{-\frac{1}{2}} \mid Y_{i}\right\}-\theta_{i} E\left\{Y_{i}^{2}\left(\sum_{j=1}^{q} \theta_{j} Y_{j}^{2}\right)^{-\frac{3}{2}} \mid Y_{i}\right\}
$$

and

$$
g_{2}^{\prime}\left(Y_{i}\right)=E\left\{\left(\sum_{j=q+1}^{q+r} \theta_{j} Y_{j}^{2}\right)^{-\frac{1}{2}} \mid Y_{i}\right\}-\theta_{i} E\left\{Y_{i}^{2}\left(\sum_{j=q+1}^{q+r} \theta_{j} Y_{j}^{2}\right)^{-\frac{3}{2}} \mid Y_{i}\right\} .
$$

For $g_{1}\left(Y_{i}\right)$, we have that:

$$
\begin{aligned}
& m_{i} E\left\{Y_{i}\left(\sum_{j=1}^{q} \theta_{j} Y_{j}^{2}\right)^{-\frac{1}{2}}\right\} \\
= & m_{i} E\left(g_{1}\left(Y_{i}\right)\right) \\
= & \left(m_{i}-2\right) E\left(Y_{i} g_{1}\left(Y_{i}\right)\right)-2 E\left(Y_{i}^{2} g^{\prime}{ }_{1}\left(Y_{i}\right)\right) \\
= & \left(m_{i}-4\right) E\left\{Y_{i}^{2}\left(\sum_{j=1}^{q} \theta_{j} Y_{j}^{2}\right)^{-\frac{1}{2}}\right\}+2 \theta_{i} E\left\{Y_{i}^{4}\left(\sum_{j=1}^{q} \theta_{j} Y_{j}^{2}\right)^{-\frac{3}{2}}\right\} .
\end{aligned}
$$

Based on the above, we see the following

$$
\begin{aligned}
& E\left\{\left(\sum_{i=1}^{q} \theta_{i} Y_{i}\right)\left(\sum_{i=1}^{q} \theta_{i} Y_{i}^{2}\right)^{-\frac{1}{2}}\right\} \\
= & \sum_{i=1}^{q} \theta_{i} E\left\{Y_{i}\left(\sum_{j=1}^{q} \theta_{j} Y_{j}^{2}\right)^{-\frac{1}{2}}\right\} \\
= & E\left\{\left(\sum_{i=1}^{q} m_{i}^{-1}\left(m_{i}-4\right) \theta_{i} Y_{i}^{2}\right)\left(\sum_{i=1}^{q} \theta_{i} Y_{i}^{2}\right)^{-\frac{1}{2}}\right\}+ \\
& 2 E\left\{\left(\sum_{i=1}^{q} m_{i}^{-1} \theta_{i}^{2} Y_{i}^{4}\right)\left(\sum_{i=1}^{q} \theta_{i} Y_{i}^{2}\right)^{-\frac{3}{2}}\right\} .
\end{aligned}
$$

From Cauchy-Schwarz inequality, we can tell:

$$
\sum_{i=1}^{q} m_{i}^{-1} \theta_{i}^{2} Y_{i}^{4} \geq\left(\sum_{i=1}^{q} m_{i}\right)^{-1}\left(\sum_{i=1}^{q} \theta_{i} Y_{i}^{2}\right)^{2} .
$$

Applying (5.3) into (5.2), we have the following:

$$
\begin{aligned}
& E\left\{\left(\sum_{i=1}^{q} \theta_{i} Y_{i}\right)\left(\sum_{i=1}^{q} \theta_{i} Y_{i}^{2}\right)^{-\frac{1}{2}}\right\} \\
\geq & E\left\{\left[\sum_{i=1}^{q}\left(m_{i}^{-1}\left(m_{i}-4\right)+2\left(\sum_{j=1}^{q} m_{j}\right)^{-1}\right) \theta_{i} Y_{i}^{2}\right]\left(\sum_{i=1}^{q} \theta_{i} Y_{i}^{2}\right)^{-\frac{1}{2}}\right\} .
\end{aligned}
$$


Similarly for $g_{2}\left(Y_{i}\right)$, we have that:

$$
\begin{aligned}
& E\left\{\left(\sum_{i=q+1}^{q+r} \theta_{i} Y_{i}\right)\left(\sum_{q+i=1}^{q+r} \theta_{i} Y_{i}^{2}\right)^{-\frac{1}{2}}\right\} \\
\geq & E\left\{\left[\sum_{i=q+1}^{q+r}\left(m_{i}^{-1}\left(m_{i}-4\right)+2\left(\sum_{j=q+1}^{q+r} m_{j}\right)^{-1}\right) \theta_{i} Y_{i}^{2}\right]\left(\sum_{i=q+1}^{q+r} \theta_{i} Y_{i}^{2}\right)^{-\frac{1}{2}}\right\} .
\end{aligned}
$$

Thus, inequality (5.1) is true if there exists $1<b<2$, such that

$$
m_{i}^{-1}\left(m_{i}-4\right)+2\left(\sum_{j=1}^{q} m_{j}\right)^{-1} \geq \frac{1}{b}
$$

for all $i=1,2, \ldots, q$, and

$$
m_{i}^{-1}\left(m_{i}-4\right)+2\left(\sum_{j=q+1}^{q+r} m_{j}\right)^{-1} \geq \frac{b}{2}
$$

for all $i=q+1, q+2, \ldots, q+r=p$.

\section{Remark 1}

Observe that inequality (5.4) and inequality (5.5) are respectively equivalent to:

$$
\left(1-\frac{1}{b}\right)\left\{m_{\min }\left(m_{\min }-\frac{2}{1-\frac{1}{b}}\right)+\left(\sum_{j=1, j \neq \min }^{q} m_{j}\right)\left(m_{\min }-\frac{4}{1-\frac{1}{b}}\right)\right\} \geq 0
$$

and

$$
\left(1-\frac{b}{2}\right)\left\{m_{\min }\left(m_{\min }-\frac{2}{1-\frac{b}{2}}\right)+\left(\sum_{j=q+1, j \neq \min }^{q+r} m_{j}\right)\left(m_{\min }-\frac{4}{1-\frac{b}{2}}\right)\right\} \geq 0 .
$$

In order to have solutions for inequalities (5.6) and (5.7), we need $1<b<2$.

\section{Remark 2}

One symmetric solution is to let $b=\sqrt{2}$. Check the Condition (5.6),

(1) When $q=1$, Condition (5.6) holds if $m_{1} \geq \frac{4}{2-\sqrt{2}} \approx 7$.

(2) When $q=2$, Condition (5.6) holds if one of the following holds:

(a) $m_{\min }=11$, and the other $11 \leq m_{j, j \neq \min } \leq 17$

(b) $m_{\min }=12$, and the other $12 \leq m_{j, j \neq \min } \leq 37$

(c) $m_{\min }=13$, and the other $13 \leq m_{j, j \neq \min } \leq 122$

(d) $m_{i} \geq 14$ for $i=1,2$.

(3) When $q=3$, Condition (5.6) holds if one of the following holds:

(a) $m_{\min }=12$, and $\sum_{j=1}^{3} m_{j, j \neq \min } \leq 37$. So either all are equal to 12 or some $m_{i}=13$ and the other two are each equal to 12 .

(b) $m_{\min }=13$, and $\sum_{j=1}^{3} m_{j, j \neq \min } \leq 122$ 
(c) $m_{i} \geq 14$ for all $i=1,2,3$.

(4) When $q=4$, Condition (5.6) holds if one of the following holds:

(a) $m_{\min }=13$, and $\sum_{j=1}^{4} m_{j, j \neq \min } \leq 122$

(b) $m_{i} \geq 14$ for all $i=1,2,3,4$.

(5) When $q=5$, Condition (5.6) holds if one of the following holds:

(a) $m_{\min }=13$, and $\sum_{j=1}^{5} m_{j, j \neq \min } \leq 122$

(b) $m_{i} \geq 14$ for all $i=1,2,3,4,5$.

(6) When $q=6$, Condition (5.6) holds if one of the following holds:

(a) $m_{\min }=13$, and $\sum_{j=1}^{6} m_{j, j \neq \min } \leq 122$

(b) $m_{i} \geq 14$ for all $i=1,2,3,4,5,6$.

(7) When $q=7$, Condition (5.6) holds if one of the following holds:

(a) $m_{\min }=13$, and $\sum_{j=1}^{7} m_{j, j \neq \min } \leq 122$

(b) $m_{i} \geq 14$ for all $i=1,2,3,4,5,6,7$.

(8) When $q=8$, Condition (5.6) holds if one of the following holds:

(a) $m_{\min }=13$, and $\sum_{j=1}^{8} m_{j, j \neq \min } \leq 122$

(b) $m_{i} \geq 14$ for all $i=1,2,3,4,5,6,7,8$.

(9) When $q=9$, Condition (5.6) holds if one of the following holds:

(a) $m_{\min }=13$, and $\sum_{j=1}^{9} m_{j, j \neq \min } \leq 122$

(b) $m_{i} \geq 14$ for all $i=1,2,3,4,5,6,7,8,9$.

(10) When $q \geq 10$, Condition (5.6) holds if $m_{i} \geq 14$ for all $i=1,2, \ldots, q$.

Similar discussion can be found for condition (5.7).

\section{Conclusion}

In this paper we compared the $p$-source based GDE with its $q$-sub-source based competitors under Pitman closeness criterion. We established a necessary condition and several sufficient conditions for the $p$-source based GDE to be Pitman closer than its $q$-sub-source based GDE. We further discussed the sample size requirement corresponding to each source, and we found that one sufficient condition is $n_{i} \geq 15$ for $i=1,2, \ldots, p$.

This sample size requirement is relatively close to the requirement based on mean square errors. Hence in our point of view, the $p$-source-based GDE dominates any other $q$-sub-source based GDE not only in terms of mean square error loss function but also in the sense of Pitman closeness criterion of probability coverage, when the sample size of each source is moderately large enough.

\section{Acknowledgements}

Research is supported by the U.S. National Science Foundation Grants DMS-1306394 and DMS1546674. This work was undertaken during regular visits of Bikas.K. Sinha at University of Illinois, Chicago. He is a retired professor of Indian Statistical Institute, Kolkata. The authors are extremely thankful to two anonymous referees for critically reading the manuscript and offering numerous suggestions towards clearer presentation of our ideas. 


\section{References}

[1] Graybill, F.A. and Deal,R.B. (1959): “Combining unbiased estimators.” Biometrics, 15, 543-550.

[2] HAfF, L.R. (1977): "Minimax estimators for a multinormal precision matrix." J. Multivar. Anal., 7(3), 374-385.

[3] Norwood, T.E and Hinkelmann, K.JR. (1977): "Estimating the common mean of several normal populations." Ann. Stat., 5, 1047-1050.

[4] KhatRI, C.G. and SHAh, K.R. (1974): "Estimation of location parameters from two linear models under normality." Commun. Stat. Theory Methods, 3, 647-663.

[5] Kubokawa, T. (1989): "Closer estimators of a common mean in the sense of Pitman." Ann. Inst. Stat. Math, 41, 477-484.

[6] Pitman, E. (1937): “The closest estimates of statistical parameters." Mathematical Proceedings of the Cambridge Philosophical Society, 33, 212-222.

[7] SARKAR, S.K. (1991): "On estimating the common mean of several normal populations under the pitman closeness criterion." Commun. Stat. Theory Methods, 20, 3487-3498.

[8] SHINOZAKI, N. (1978): "A note on estimating the common mean of $\mathrm{k}$ normal distributions and the stein problem.” Commun. Stat. Theory Methods, 7, 1421-1432. 